\title{
Influences of geometric and dynamic synchronous errors onto machined surface in 5-axis machining center
}

\author{
Shogo HASEGAWA*, Ryuta SATO* and Keiichi SHIRASE* \\ *Department of Mechanical Engineering, Kobe University, \\ 1-1 Rokko-dai, Nada, Kobe 657-8501, Japan \\ E-mail: sato@mech.kobe-u.ac.jp
}

Received 28 February 2016

\begin{abstract}
Unexpected glitches typically occur on the finished surface machined by the 5 -axis machining centers, because of geometric and dynamic synchronous errors of the machine. In this study, actual ball-nosed end milling tests of hemispheres and its finished surface simulations with different geometric errors and different position loop gain of feed drive systems were carried out, in order to clarify the influence of the geometric and dynamic synchronous errors onto machined surface. As the results, it is clarified that the influence of geometric errors onto the machined surface is depending on the relationships between the movement of the axes and the surface geometry. In addition, the dynamic synchronous error also influences the machined surface when the velocity of translational and rotary axes changed rapidly.
\end{abstract}

Key words : 5-axis machining center, Geometric error, Dynamic synchronous error, Machined surface, Simulation

\section{Introduction}

The 5-axis machining centers can control both of relative position and angle between a tool and a workpiece, are generally applied to machine mechanical and aero dynamics parts having complex shapes such as impellers. The 5-axis machining centers also applied to machine molds and dies with ball-nosed end mill, because the lead and tilt angles of the tool can be controlled by using the rotary axes in order to avoid the tool tip point of ball-nosed end mill which has zero circumferential velocity. The 5-axis machining centers have many advantages as mentioned above, but they also have disadvantages. One of the most essential disadvantages is the motion accuracy. Because the 5-axis machining centers have many error sources than the conventional three-axis machining centers.

Rotary axes of the 5-axis machining centers have position and orientation errors of the rotational centers, called geometric errors (ISO 230-1, 2012). Since it is known that the geometric errors much deteriorate the geometrical accuracy of the machined parts, several research works have been carried out to identify and compensate the errors. Tsutsumi and Saito (Tsutsumi and Saito, 2003) proposed an identification method for the geometric errors using a ball-bar system. Bringmann and Knapp (Bringmann and Knapp, 2006) also proposed the evaluation method by using a reference sphere and displacement sensors called "R-test". Compensation of the errors based on the identified results is also be possible (Tsutsumi et al., 2013). Evaluation method for the geometric errors through the actual cutting tests is also proposed. Ibaraki et al. proposed the machining patterns with square end mill (Ibaraki et al., 2010 and 2014), and the method is also investigated by the other researchers (Velenosi et al., 2015). Another error source in the machining is the dynamic synchronous errors between translational and rotary axes. An evaluation method for the dynamic synchronous accuracy had been proposed (Tsutsumi et al., 2007). One of the authors also investigated the evaluation and improvement methods for the dynamic synchronous accuracy (Sato and Tsutsumi, 2007 and 2011).

It is also known that the unexpected problems may occur on the finished surface by the 5 -axis machining centers, because of the geometric and dynamic synchronous errors in the actual manufacturing field. Since the various error sources exist in the shape generation process, it is difficult to solve the problem after the actual cutting test. Consequently, the problems on the finished surface should be predicted by a simulation, and the causes of the problem should be solved before the actual cutting. From this point of view, the authors developed a machined surface simulator 
consists of the geometrical and dynamic synchronous error models (Sato et al., 2014). In addition, it had already known that the the mechanical vibrations of the machine tool structure (Kim and Kim, 2011), tool deformations due to the cutting force (Kakino et al., 2000, Tanaka et al., 2008, Buj-Corral et al., 2012), and geometrical accuracy of the tool (Hirogaki et al., 2007) affects the machined surface. However, the relationships between the each geometrical and dynamic error factors of machine tools and the machined surface have not been clarified.

The purpose of this study is to clarify the relationships between the each geometrical and dynamic error factors of machine tools and the machined surface. In this study, actual ball-nosed end milling tests of hemispheres and its finished surface simulations considering the each geometric error and different position loop gain of feed drive systems which is a parameter affecting the dynamic synchronous accuracy were carried out, in order to clarify the influence of the each error factors onto machined surface.

\section{Geometrical Errors and Feed Drive System Model}

All ball-nosed end milling tests in this study were carried out using a vertical type 5-axis machining center NMV1500DCG (DMG MORI SEIKI Co. Ltd.) having two rotary axes (B-axis and C-axis) in the table, as shown in Figure 1. Translational axes are driven by ball-screws, and rotary axes are driven by Direct Drive (DD) motors. Y- and Z-axis are driven by two AC servo motors and ball-screws, respectively. Translational axes are controlled by semi-closed control system based on the rotational angle of the motors.

Geometric error is mainly caused by the assembling errors. According to theory of the previous study (Tsutsumi and Saito, 2003), it is necessary to consider 8 geometric errors (4 positional and 4 orientation errors) of average rotational center line of the rotary axes in this machining center as listed in Table 1. Numerically controlled machine tools actuate feed drive systems of each axis following positional commands generated from NC-program, and perform the intended profiling. Feed drive systems consist of feed drive mechanism and control system (position, velocity and current control system). In this study, the simplified model of feed drive system considered only position control system as shown in Figure 2 was used for machining simulation and reproduce dynamic behavior, because the velocity and current control system response is much faster than the position control system and negligible. In Figure $2, G_{p}$ is the position loop which represents the response of the feed drive system, $X_{\text {ref }}$ and $X_{t}$ are the position commands and outputs.

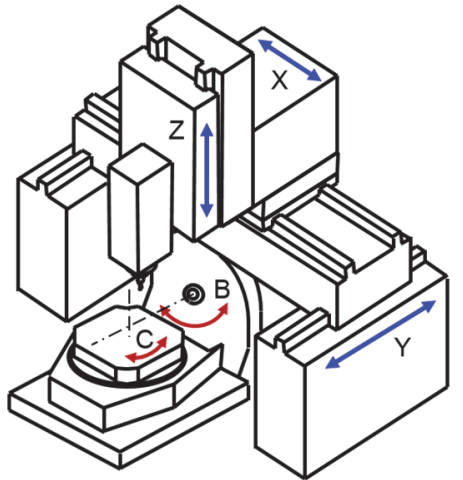

Fig. 1 Five-axis machining center

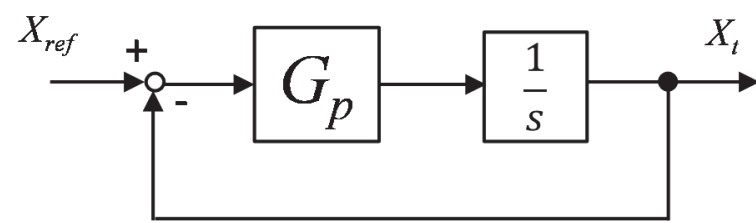

Fig. 2 Simplified block diagram of feed drive system

Table 1 Geometric errors in rotary axes

\begin{tabular}{c|c}
\hline Symbol & Error \\
\hline$\delta_{x B Y}$ & Positional error of B-axis in X-direction with respect to Y-axis \\
\hline$\delta_{y B Y}$ & Positional error of B-axis in Y-direction with respect to Y-axis \\
\hline$\delta_{z B Y}$ & Positional error of B-axis in Z-direction with respect to Y-axis \\
\hline$\delta_{x C B}$ & Offset of C-axis from B-axis in X-direction with respect to B-axis \\
\hline$\alpha_{B Y}$ & Orientation error of B-axis around X-axis with respect to Y-axis \\
\hline$\alpha_{C B}$ & Orientation error of C-axis around X-axis with respect to B-axis \\
\hline$\beta_{B Y}$ & Orientation error of B-axis at B-axis origin \\
\hline$\gamma_{B Y}$ & Orientation error of B-axis around Z-axis with respect to Y-axis \\
\hline
\end{tabular}




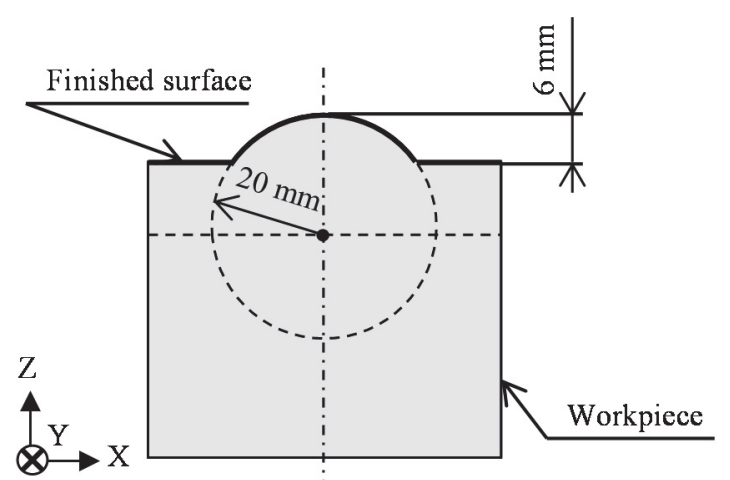

(a) Workpiece

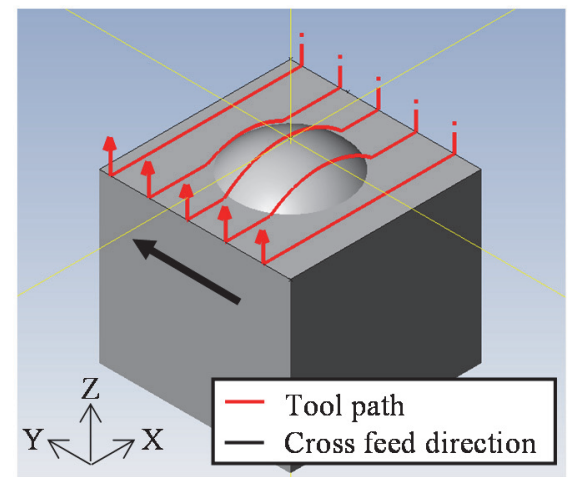

(b) Tool path

Fig. 3 Workpiece and tool path

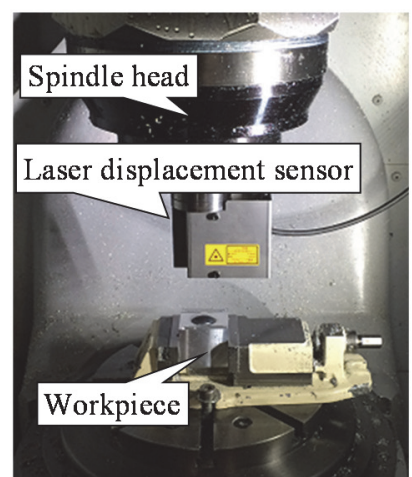

(a) Machined shape measurement

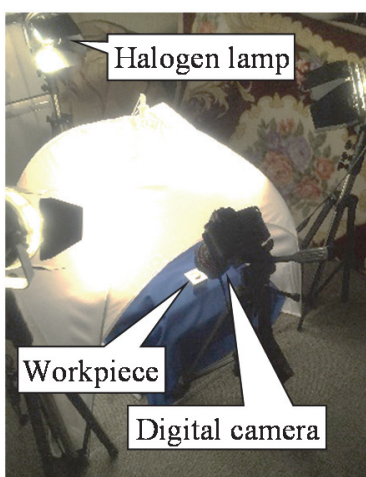

(b) Setup for taking photograph

Table 2 Cutting conditions

\begin{tabular}{c|c|c}
\hline & Condition A & Condition B \\
\hline Tool shape & \multicolumn{2}{|c}{$\phi 6$ Ball-end mill } \\
\hline Number of flutes & \multicolumn{2}{|c}{2} \\
\hline Workpicec & Aluminum alloy (A5052P) \\
\hline Feed rate & $60 \mathrm{~mm} / \mathrm{min}$ & $2000 \mathrm{~mm} / \mathrm{min}$ \\
\hline Spindle speed & $600 \mathrm{~min}^{-1}$ & $20000 \mathrm{~min}^{-1}$ \\
\hline Axial depth of cut & $0.5 \mathrm{~mm}$ & $1 \mathrm{~mm}$ \\
\hline Radial depth of cut & $0.2 \mathrm{~mm}$ & $0.05 \mathrm{~mm}$ \\
\hline
\end{tabular}

It is known that the motion errors around quadrant changes due to the friction forces makes motion errors (Sato, 2012). One of the authors also investigated the phenomena around motion direction changes and the influence of the error onto the finished surface (Nishio et al., 2012). On the other hand, the purpose of this study is to clarify the geometric and dynamic synchronous errors between the axes onto the machined surface by 5-axis machining. From this point of view, the feed drive system model is simplified to only express the dynamic synchronous errors. The authors confirmed that the simplified model only consider the position loop gain can enough can express the dynamic synchronous accuracy between the axes. It is also confirmed that the motion error around quadrant changes is not dominant in the 5-axis machining in this study.

\section{Machining Tests and Simulations}

\subsection{Machining Tests and Measurement}

Figure 3 (a) shows the geometric shape designed for the end-milling test. The workpiece had the upper part $6 \mathrm{~mm}$ of a sphere with $20 \mathrm{~mm}$ radius. The tool path was the uni-directional feed of the scanning line along the X-axis, as 
shown in Figure 3 (b). In the milling test, the tilt angle (roll angle in the feed direction) of tool orientation was set to zero, and the lead angle (pitch angle in the feed direction) was set to $20^{\circ}$ by 5 -axis simultaneous control machining. Although it is known that the geometrical accuracy of the ball-nosed end mill affects the machined surface (Hirogaki et al., 2007), it is expected that the tool shape accuracy does not have significant influence because the geometrical relationship between the tool and machined surface keeps constant during the machining of spherical part.

The finished surfaces were measured by using a laser-displacement sensor as shown in Figure 4 (a). The result of on-machine measurement includes the influence of motion error. In this study, since the result of 5-axis simultaneous control machining was measured by using only translational axes, the measuring result did not include the influence of the motion of rotary axes. The finished surface was illuminated with three halogen lamps through white cloth, in order to avoid reflection (Figure 4 (b)).

Table 2 lists the cutting conditions. The condition A was used in order to investigate influence of geometric errors and set feed rate lower so as to eliminate influence of dynamic synchronous errors. On the other hand, the condition B was used to investigate influence of dynamic synchronous errors and set feed rate higher. NC program for the condition A is generated based on the coordinate transformation approach without TCP control function. NC program for the condition B is generated by using a CAM system (ESPRIT, DP Technology Corporation). Tolerance was set to $10 \mu \mathrm{m}$ and TCP control function is applied for the machining. Both of the program consists of only the linear interpolation (G01). It is confirmed that the generated NC programs have enough accuracy thorough the simulated machined shapes based on the NC programs and positional commands for each axis.

A Ball-nosed end mill used in this study is commercial DLC coated end mill (DLC-2MB, Mitsubishi Materials Corporation). Shapeless of the cutting edge (cutting edge radius) is between 2 and $4 \mu \mathrm{m}$. Semi-finishing and finishing processes are carried out continuously without tool change. Condition of the cutting edge was checked by a micro scope and it is confirmed that there is no significant wear and breakage of the cutting edges. In addition, machined shapes of the semi-finishing process have 0.5 or $1 \mathrm{~mm}$ larger radius than the final machined shape. As the result, it is expected that the removal rate during the finishing process of the spherical part becomes constant.

Since the vertical 5-axis machining center used in this study had higher geometric accuracy, influence of the geometric errors to the finished surface was negligible. In this study, therefore, NC program was generated as if geometric errors exist in order to exaggerate their influence. The position loop gain of feed drive systems was also set lower deliberately in order to exaggerate influence of dynamic synchronous errors.

\subsection{Simulation Method}

Figure 5 shows the flow chart of the simulation method proposed by the authors (Sato et al., 2014). In this method, both of effects of geometric errors between the axes and dynamic behavior of feed drive systems can be considered. Although it was known that the mechanical vibrations of the machine tool structure (Kim and Kim, 2011), tool deformations due to the cutting force (Kakino et al., 2000, Tanaka et al., 2008, Buj-Corral et al., 2012), and geometrical accuracy of the tool (Hirogaki et al., 2007) affects the machined surface, those factors were not considered in order to clarify the influence of motion errors only.

In the first step of the proposed method, position and angle commands for each axis during the cutting motion (air cut) are acquired from the numerical controller (NC) of the machine. These inputs can be collected using either the internal monitoring functions of the NC (FANUC 30i) or an external PC equipped with connection software. Acceleration-deceleration filters and error compensators are implemented in the NC. In the second step, the dynamic response behaviors to the commands of each axis are simulated by the mathematical model of each feed drive system by the simplified model shown in Figure 2. This model only considers the servo delay of the position control loop.

In order to simulate the shape of the machined surface, a coordinate transformation between the machine and table coordinate systems is required. Obtained output in the machine coordinate system converted into tool position and orientation in the table coordinate system using a homogeneous transformation matrix considered effects of geometric errors. The origin of the machine coordinate system is the reference position or angle of each axis, and the coordinate system is fixed on the machine main frame. The table coordinate system is defined as the coordinate system that is rotated with the $\mathrm{C}$ - and $\mathrm{B}$-axis rotation. The $\mathrm{X}$ and $\mathrm{Y}$ coordinate origins are at the rotational center of the $\mathrm{C}$-axis, and a $\mathrm{Z}$ coordinate origin is at the rotational center of the B-axis. The position of the translational axes in the machine coordinate system ${ }^{M} \boldsymbol{X}$ can be transferred to the position in the table coordinate system ${ }^{T} \boldsymbol{X}$ as follows.

$$
{ }^{T} \boldsymbol{X}=D(-C) D\left(-\alpha_{C B}\right) D\left(\delta_{x C B}\right) D(-B) D\left(-\gamma_{B Y}\right) D\left(-\alpha_{B Y}\right){ }^{M} \boldsymbol{X}
$$




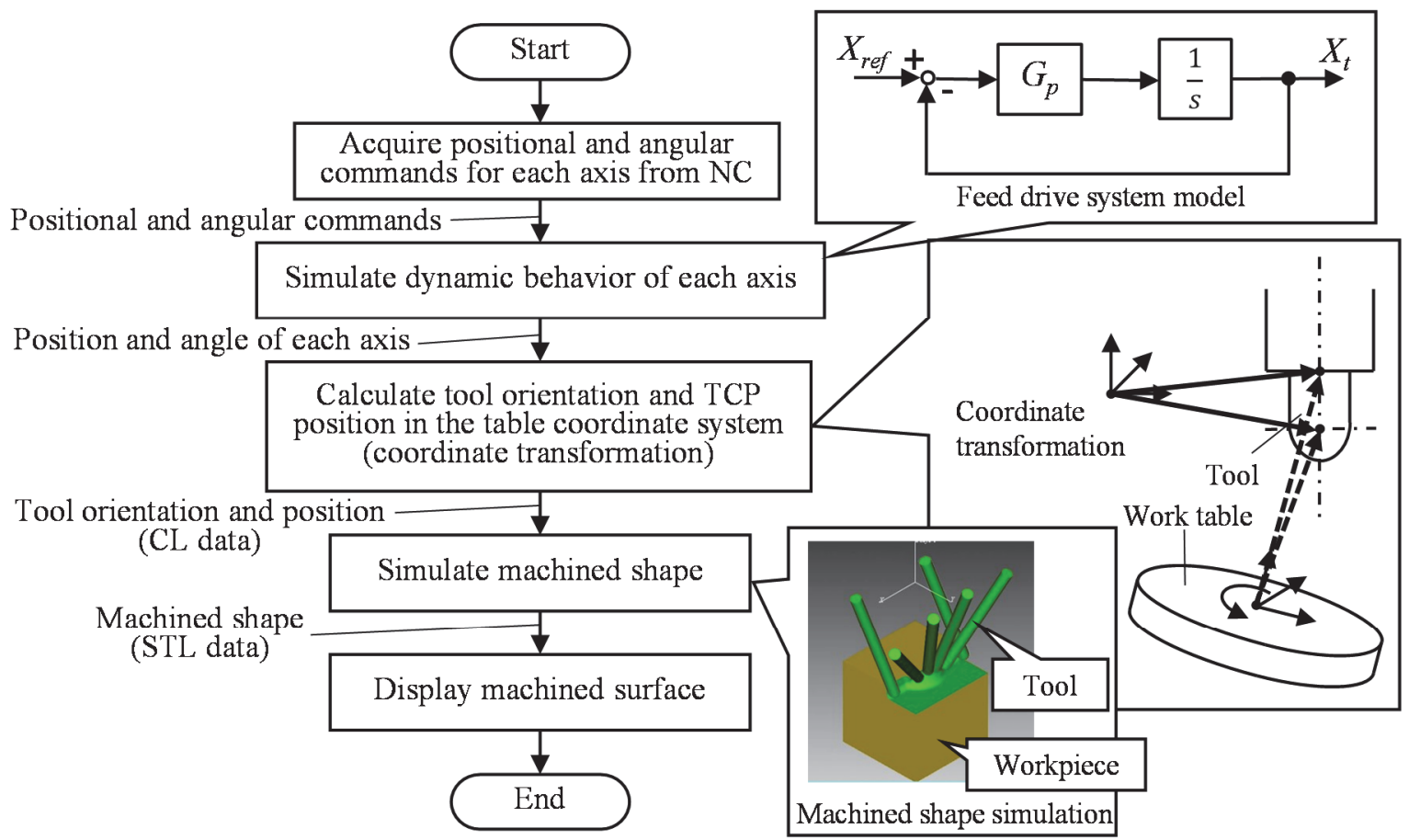

Fig. 5 Simulation flow

where, $B$ and $C$ are the rotational angle of the $\mathrm{B}$ - and C-axis, respectively. $D$ is the coordinate transform matrixes. It is possible to obtain the position in the table coordinate system ${ }^{T} \boldsymbol{X}$ for both the TCP and spindle nose, and the tool orientation vector in the table coordinate system can be obtained from the positions.

As the result, tool orientation and position for every time steps (CL data) can be obtained. In the next step of the proposed method, the shape of the machined surface is simulated based on the CL data. In this study, VERICUT ${ }^{\circledR}$ software (CGTech Co., Ltd.) was used for this simulation. Although VERICUT ${ }^{\circledR}$, which is widely used to simulate the machined shape, mainly focuses on checking a new NC program before new code is run, it is also possible to achieve more precise simulations by setting the resolution parameters as small as possible. The simulated shape is converted to the Standard Triangulated Language (STL) data and rendered by the rendering software; Blender (Blender foundation).

\section{Influence of Geometric Errors}

\subsection{Influence of Positional Errors}

Figure 6 shows the simulated finished shapes and surfaces in case of $\delta_{y B Y}$ is set as $0.1 \mathrm{~mm}$ (Figure 6 (a)) and in case of $\delta_{z B Y}$ is set as $0.1 \mathrm{~mm}$ (Figure $6(\mathrm{~b})$ ). Figure 7 shows the actual finished shapes and surfaces, under the same conditions of the simulations. "Shape" means the geometry of the machined surface, and "Surface" means the appearance of that in this study. In the figures, the radial deviation is enlarged to 50 times to clearly show the influence of the errors. It can be seen from the figures that a dent on the right side of the finished shape exists and it is able to see on the finished surface, in case of $\delta_{y B Y}$ is set as $0.1 \mathrm{~mm}$, on the other hand, there is no dent on the machined surface and shape, in case of $\delta_{z B Y}$ is set as $0.1 \mathrm{~mm}$. It is clear from the results that the influence of geometric errors onto the finished surface depends on the relationships between the direction of the errors and the surface. These phenomena can be simulated accurately. This fact suggests that the geometry of the machined shape is mainly determined by the geometrical accuracy of the machine tools in this machining condition. Influence of the other error sources, such as mechanical vibration of the machine and tool, rotational accuracy of the spindle, cutting force, are negligible.

Figure 8 shows the simulated motion trajectory of a scanning line near the dentin the machine coordinate system. As can be seen from the figure, table (C-axis) is rotated $180^{\circ}$ and translational axis moved following rotational movement. The motion trajectory with $\delta_{y B Y}$ in the table coordinate system is shown in Figure 9 (a), and that with $\delta_{z B Y}$ is also shown in Figure 9 (b). As shown in Figure 8, C-axis rotated $180^{\circ}$ in the path. The motion trajectory in the table coordinate system is shifted to $+\mathrm{Y}$ direction in case of $\delta_{y B Y}$ exists as shown in Figure 9 (a), because rotational center position deviated from neutral position to $+\mathrm{Y}$ direction. 

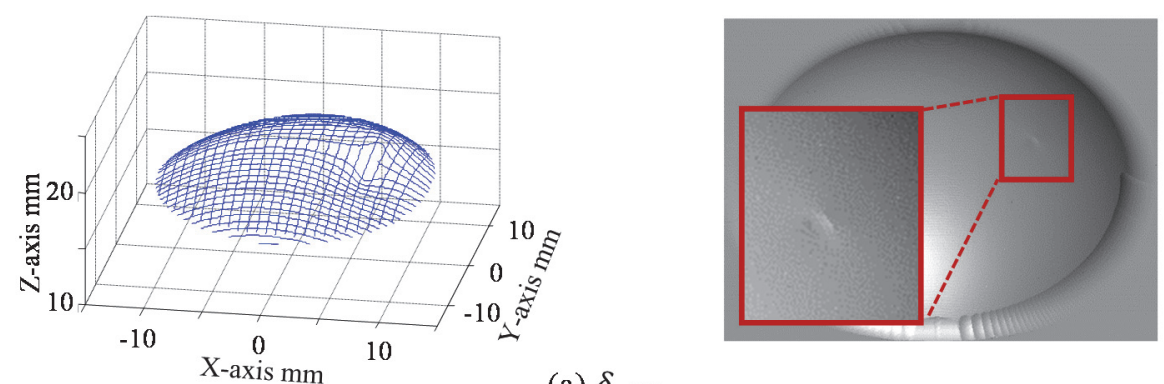

(a) $\delta_{y B Y}$
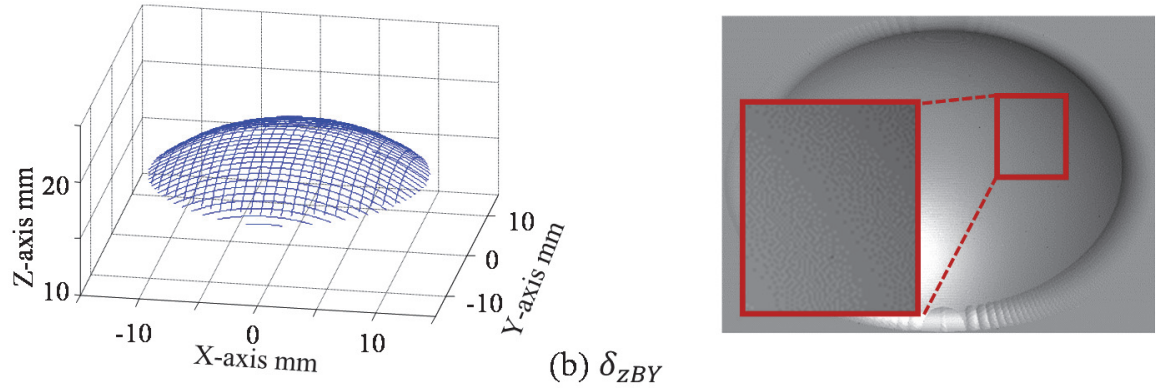

Fig. 6 Comparison of influence of geometrical errors (Simulation)
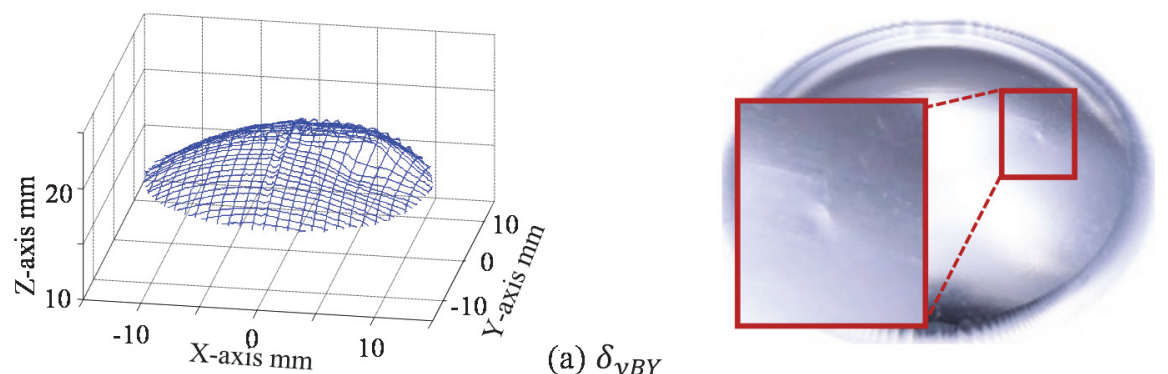

(a) $\delta_{y B Y}$
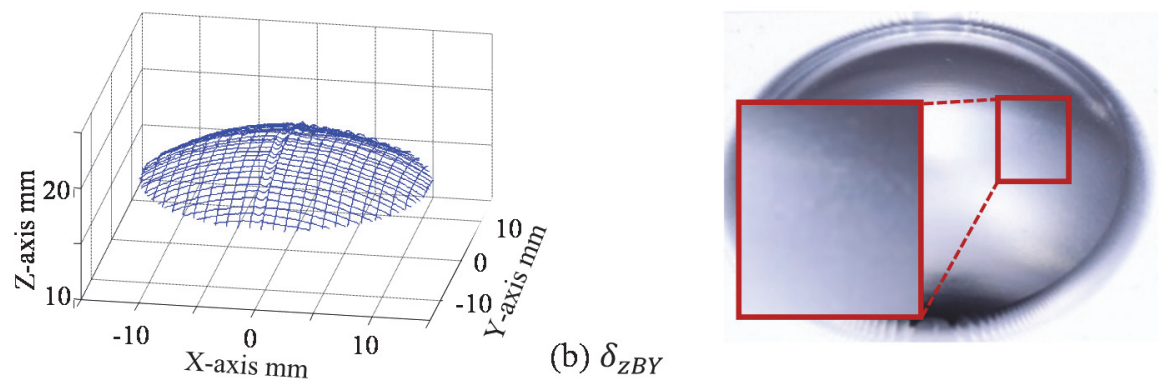

Fig. 7 Comparison of influence of geometrical errors (Experiment)

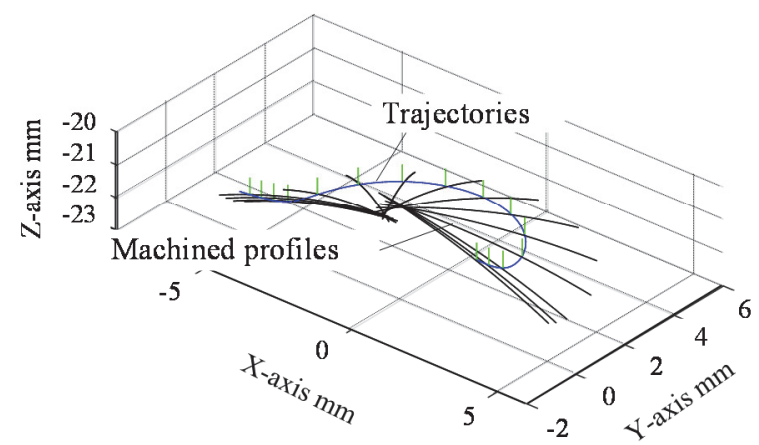

Fig. 8 Motion trajectory of scanning line in machine coordinate system 

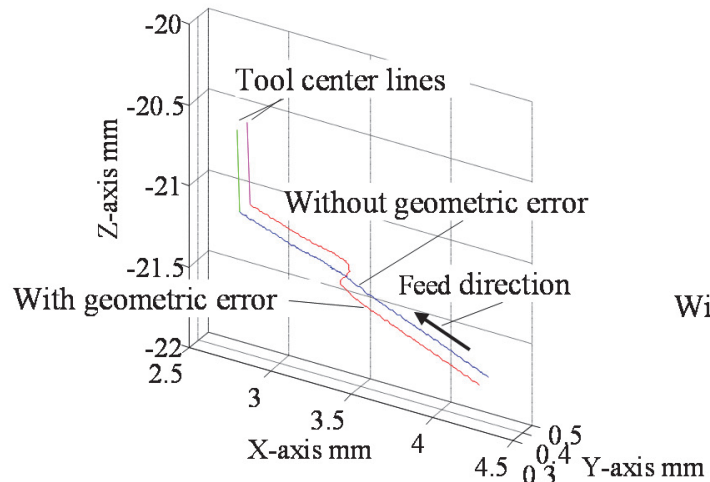

(a) $\delta_{y B Y}$

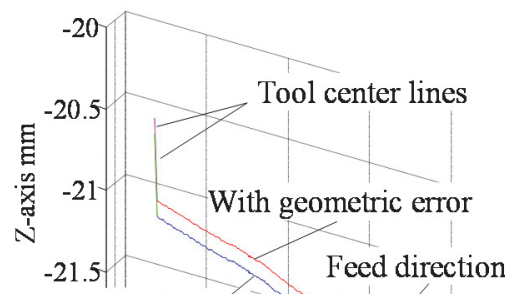

Without geometric error

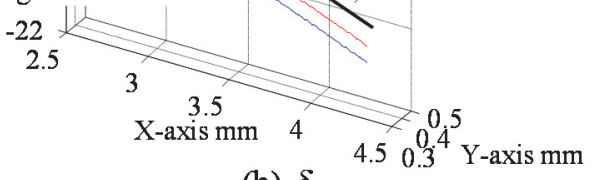

(b) $\delta_{z B Y}$

Fig. 9 Influence of positional errors on motion trajectory in table coordinate system
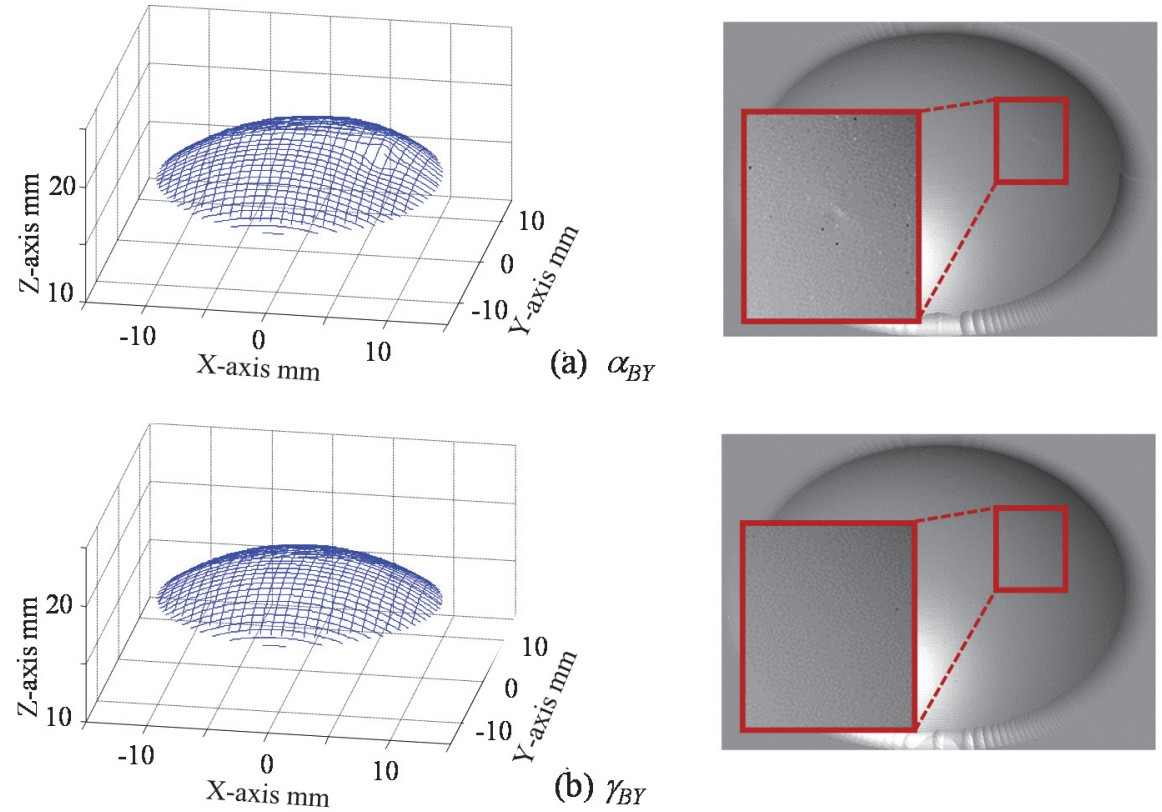

Fig. 10 Comparison of influence of geometrical errors (Simulation)
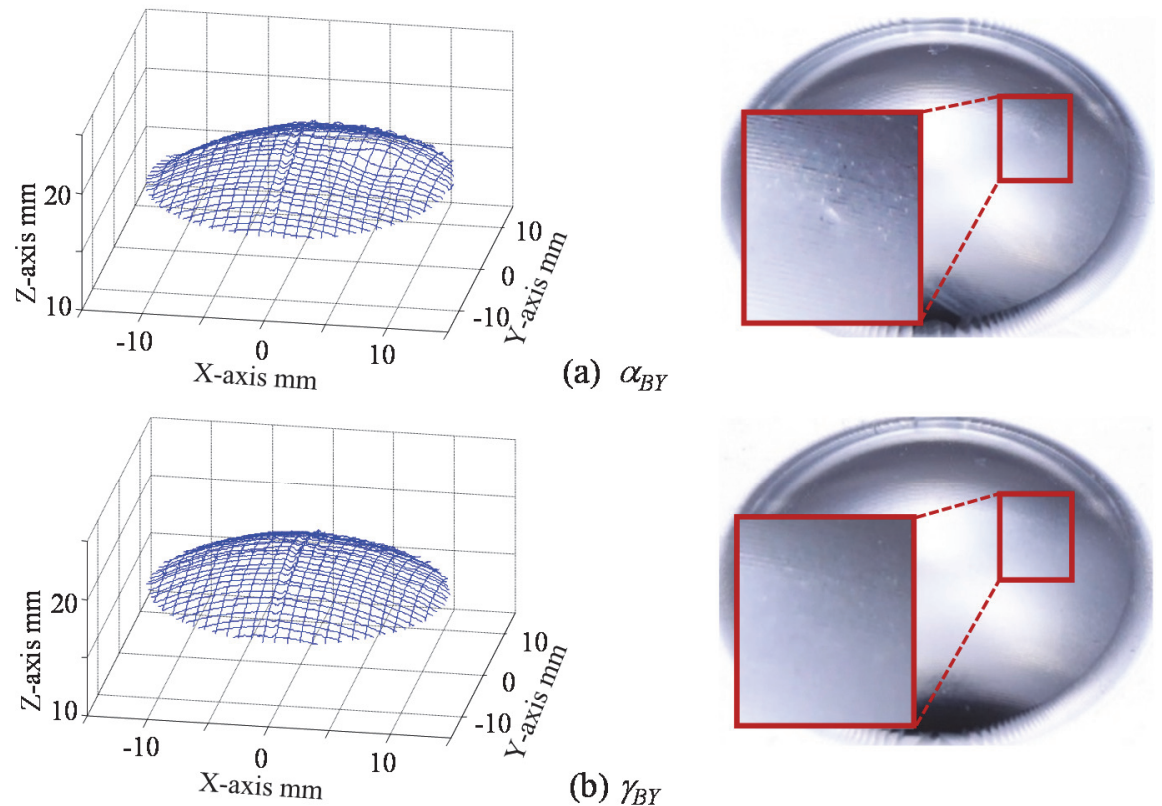

(a) $\alpha_{B Y}$

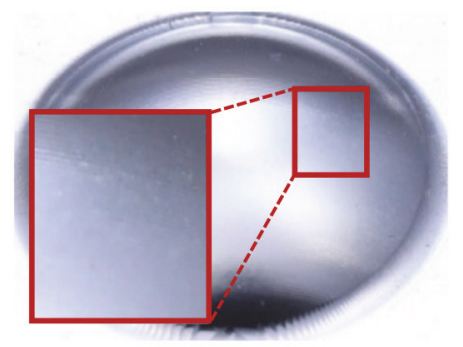

(b) $\gamma_{B Y}$

Fig. 11 Comparison of influence of geometrical errors (Experiment) 


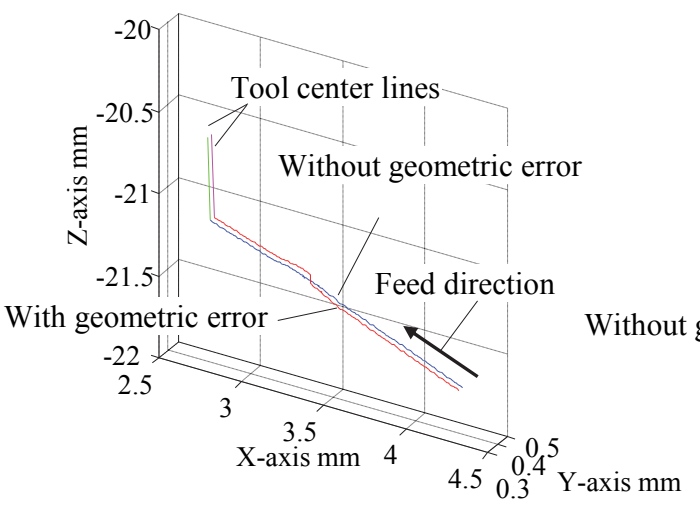

(a) $\alpha_{B Y}$

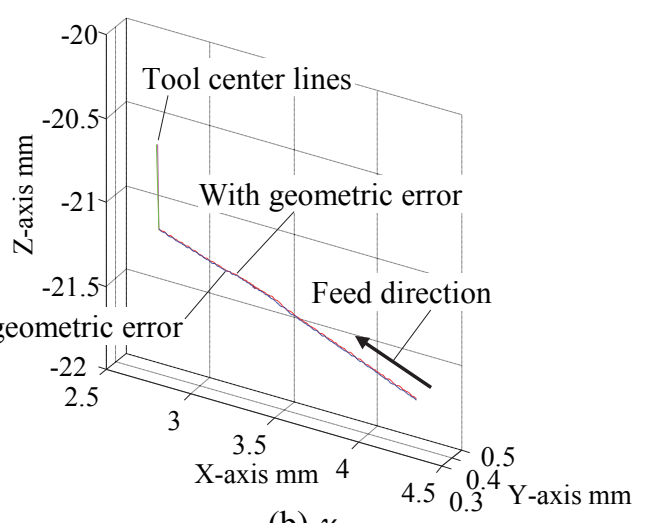

(b) $\gamma_{B Y}$

Fig. 12 Influence of orientation errors on motion trajectory in table coordinate system

This movement yielded the dent on the machined surface. On the other hand, although the deviation along Z-axis direction $\delta_{z B Y}$ yielded tracking error in the table coordinate system as shown in Figure 9 (b), the trajectory does not have step-like error.

\subsection{Influence of Orientation Errors}

Figure 10 shows the simulated shapes and surface in case of $\alpha_{B Y}$ is set as $0.1^{\circ}$ (Figure 10 (a)) and in case of $\gamma_{B Y}$ is set as $0.1^{\circ}$ (Figure $10(\mathrm{~b})$ ). Figure 11 shows the actual finished shapes and surfaces, under the same conditions of the simulations. A similar dent on the surface can be observed in case of the orientation error around X-axis, $\alpha_{B Y}$ exists, and the dent cannot be observed on the machined surface and shape in case of orientation error around Z-axis, $\gamma_{B Y}$ exists.

The motion trajectories in the table coordinate system with the orientation errors $\alpha_{B Y}$ and $\gamma_{B Y}$ are shown in Figure 12. It can be seen from the figures, in case of the orientation error around $\mathrm{X}$-axis, $\alpha_{B Y}$ exists, the motion trajectory shifted to $+Y$ direction in the table coordinate system, correspond to the influence of $\delta_{y B Y}$. On the other hand, the orientation error around $\mathrm{Z}$-axis $\gamma_{B Y}$ does not have significant influence on the motion trajectory in the case of this milling test. Because of that the table rotated horizontally in this case.

It is confirmed from the results that the influence of geometrical errors depend on the shape of the workpiece and the tool path for the milling, and the geometric errors in same direction yield similar effect onto finished surface.

\section{Influence of Dynamic Synchronous Errors}

In order to clarify the influence of the dynamic synchronous errors onto the finished surface, milling tests and simulations were carried out with different position loop gain $G_{p}(1201 / \mathrm{s}, 601 / \mathrm{s}, 301 / \mathrm{s})$, which governs tracking performance of feed drive systems. Figures 13 (a) to (c) show the results of simulations and milling tests in case of $G_{p}$ was set as $120 \mathrm{l} / \mathrm{s}$ (default), $60 \mathrm{l} / \mathrm{s}$, and $30 \mathrm{l} / \mathrm{s}$, respectively. Lower position loop gain makes larger tracking errors, and it makes larger synchronous errors. According to figures, the dent on the surface can be observed on the left side of the machined shape, especially in the results with lower position loop gain $G_{p}$.

Figure 14 shows the velocity of each axis in a scanning line of the milling motion. It is clear from the figure that simultaneous 5 -axis motion is required in the machining, and the velocities of the axes are greatly changed in the motion. The dent observed on the surface is generated around $3 \mathrm{~s}$ in the time domain in Figure 14. Around three seconds in the figures, it can be seen that the velocity of $\mathrm{Y}$ - and $\mathrm{C}$-axis changes rapidly (marked $\mathrm{A}$ in the figures). It is known that the rapid change of the velocity causes larger tracking error of the servo system, and the tracking error makes larger synchronous errors. It is clear from Figures 13 and 14, a dent observed on the surface was due to the influence of dynamic synchronous errors because lower position loop gain makes clearer dent on the surface.

It can be confirmed from the results that the influence of the dynamic synchronous error can be observed on the surface when the velocities of the axes change rapidly, and the influence of the dynamic synchronous error onto the machined surface becomes larger when lower position loop gain is applied. In addition, it can also be seen from Figure 13 that the simulated machined surfaces are not completely same with the actual ones. This fact suggests that the other error sources such as such as mechanical vibration of the machine and tool, rotational accuracy of the spindle, cutting force might have influences onto the machined surfaces in this condition. The authors need to investigate the influence 

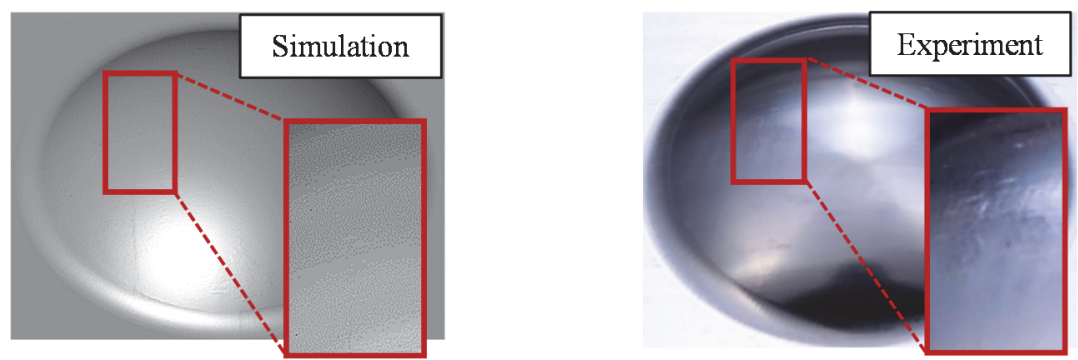

(a) $G_{p}=1201 / \mathrm{s}$
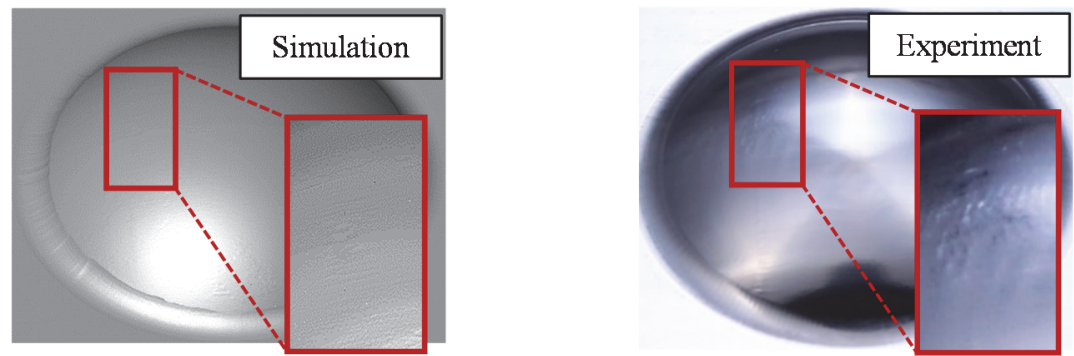

(b) $G_{p}=601 / \mathrm{s}$
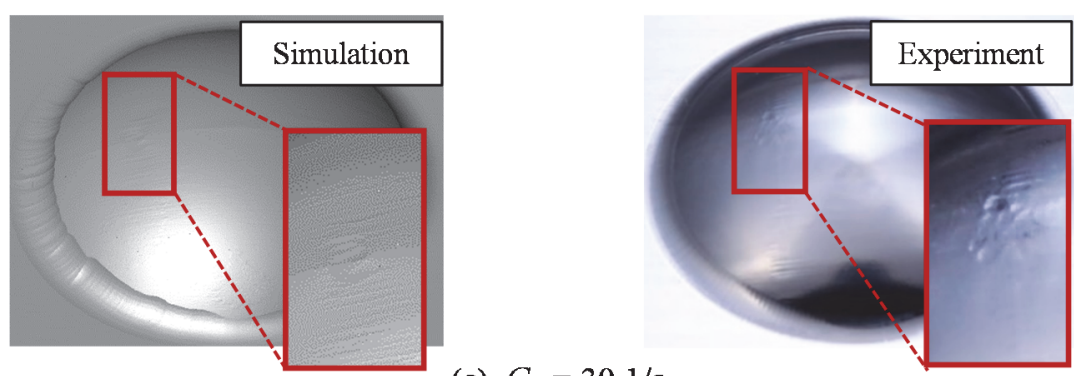

(c) $G_{p}=30 \mathrm{l} / \mathrm{s}$

Fig. 13 Influence of dynamic synchronous accuracy

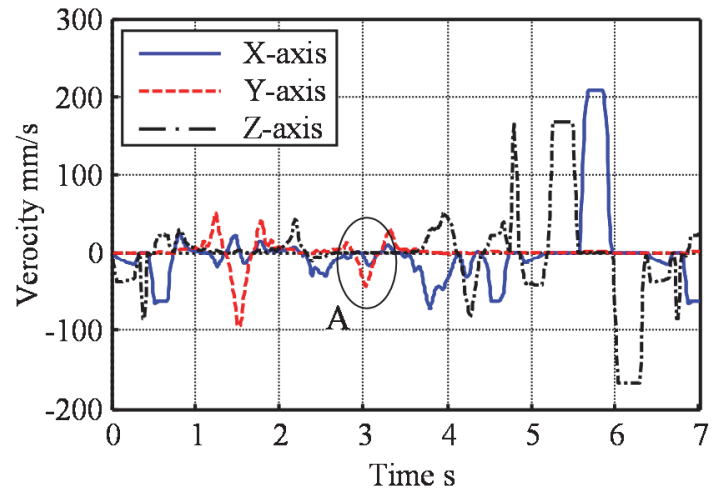

(a) Translational axes

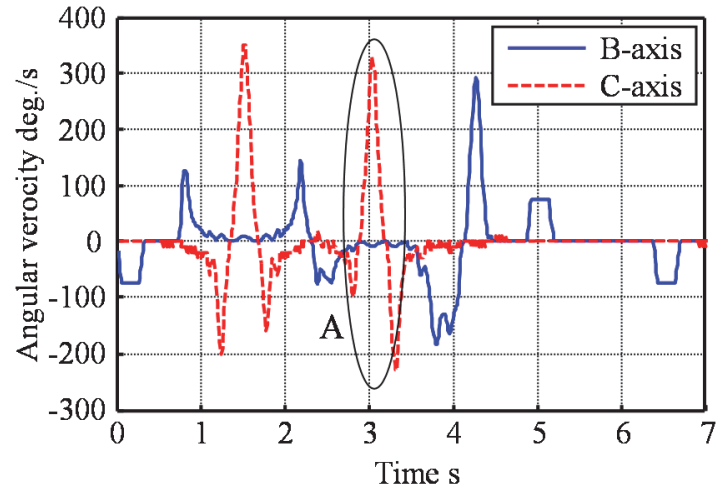

(b) Rotary axes

Fig. 14 Velocity of each axis in scanning path with simultaneous five-axis motion

of the other error sources in addition to the influence of the motion errors.

\section{Conclusion}

This study has carried out actual ball-nosed end milling tests and its simulations with the each geometric error and different position loop gain of feed drive systems in order to clarify the influence of the errors onto finished surface. Conclusions can be summarizes as follows:

1) Influence of geometrical errors depend on the shape of the workpiece and the tool path in the milling. 
2) The geometric errors in same direction yield similar effect onto finished surface.

3) Influence of the dynamic synchronous error can be observed on the finished surface when the velocity of the axes changes rapidly.

4) Influence of the dynamic synchronous error onto the finished surface becomes larger when lower position loop gain is applied.

5) The influence of the geometrical and dynamic synchronous errors onto the finished surface can accurately be simulated by the proposed method.

It is expected that the cause of the problems on the finished surface can be identified based on the analyzed finished surface. It is also expected that the results in this study can be an effective tool to achieve the intelligent virtual manufacturing which can realize the future zero-defect manufacturing systems.

\section{Acknowledgement}

This work was supported by JSPE KAKENHI (Grant-in Aid for Scientific Research (C)) Grant Number 15K05724. The authors would like to sincerely acknowledge all support from the MTTRF (Machine Tool Technologies Research Foundation).

\section{References}

Bringmann, B. and Knapp, W., Model-based 'Chace-the-ball' Calibration of a 5-axis Machining Center, CIRP annals manufacturing technology, Vol.55-1, (2006), pp.431-534.

Corral, I. B., Calvet, J. V. and Fernandez, A. D., Surface Topography in Ball-end Milling Processes as a Function of Feed Per Tooth and Radial Depth of Cut, International Journal of Machine Tools \& Manufacture, Vol. 53, (2012), pp.151-159.

Hirogaki, T., Aoyama, E., Machinaka, R., Sueda, H. and Ogawa, K., Investigation on Ball Nosed End-milled Cylindrical Surface with a Scanning Line Cutter Path, Journal of Japan Society for Precision Engineering, Vol.73, No.1, (2007), pp.96-101 (in Japanese).

Ibaraki, S., Sawada, M., Matsubara, A. and Matsushita, T., Machining Tests to Identify Kinematic Errors on Five-axis Machine Tools, Precision Engineering, Vol.34, No.3, (2010), pp.387-398.

Ibaraki, S. and Ota, Y., A Machining Test to Calibrate Rotary Axis Error Motions of Five-axis Machine Tools and its Application to Thermal Deformation Test, International Journal of Machine Tools \& Manufacture, Vol.86, (2014), pp.81-88.

ISO230-1, Test Code for Machine Tools - Part 1: Geometric Accuracy of Machines Operating under No-load or Quasi-static Conditions, (2012).

Kakino, Y., Ohtsuka, H., Nakagawa, H., Hirogaki, T. and Sasaki, M., A Study on Endmilling of Hardened Steel (lst Report), Journal of Japan Society for Precision Engineering, Vol.66, No.5, (2000), pp.730-734 (in Japanese).

Kim, J. and Kim, C., Influence of Machine Vibration on Surface Generation in Ultra Precision Machining, Proceedings of the ICOMM, No. 86, (2011), pp.497-501.

Nishio, K., Sato, R., and Shirase, K., Influence of Motion Error of Feed Drive Systems on Machined Surface, Journal of Advanced Mechanical Design, Systems, and Manufacturing, Vol.6, No.6, (2012), pp.781-791.

Sato, R. and Tsutsumi, M., High Performance Motion Control of Rotary Table for 5-axis Machining Centers, International Journal of Automation Technology, Vol.1, No.2, (2007), pp.113-119.

Sato, R. and Tsutsumi, M., Dynamic Synchronous Accuracy of Translational and Rotary Axes, International Journal of Mechatronics and Manufacturing Systems, Vol.4, No.3/4, (2011), pp.201-219.

Sato, R., Generation Mechanism of Quadrant Glitches and Its Compensation of Feed Drive Systems for NC Machine Tools, International Journal of Automation Technology, Vol.6, No.2, (2012), pp.154-162.

Sato, R., Sato, Y., Shirase, K., Campatelli, G. and Scippa, A., Finished Surface Simulation Method to Predicting the Effects of Machine Tool Motion Errors, International Journal of Automation Technology, Vol.8, No.6, (2014), pp.801-810.

Tanaka, H., Lu, L., Sato, M., and Ikua, W. B., Study on Ball End Milling of Cylindrical Surface, Transactions of Japan Society of Mechanical Engineers, Series C, Vol.74, No.744, (2008), pp.2072-2078 (in Japanese).

Tsutsumi, M. and Saito, A., Identification and Compensation of Systematic Deviations Particular to 5-axis Machining Centers, International Journal of Machine Tools \& Manufacture, Vol.43, (2003), pp.771-780. 
Tsutsumi, M., Yumiza, D., Utsumi, K. and Sato, R., Evaluation of Synchronous Motion in Five-axis Machining Centers with a Tilting Rotary Table, Journal of Advanced Design, Systems, and Manufacturing, Vol.1, No.1, (2007), pp.24-35.

Tsutsumi, M., Tone, S., Kato, N. and Sato, R., Enhancement of Geometric Accuracy of Five-axis Machining Centers based on Identification and Compensation of Geometric Deviations, International Journal of Machine Tools \& Manufacture, Vol.68, (2013), pp.11-20.

Velenosi, A., Campatelli, G. and Scippa, A., Axis Geometrical Errors Analysis through a Performance Test to Evaluate Kinematic Error in a Five Axis Tilting-rotary Table Machine Tool, Precision Engineering, Vol.39, (2015), pp.224-233.

Website: http://www.cgtech.com/

Website: http://www.blender.org/ 